\title{
L'INFLUENCE DIRECTE DU VERRAT SUR LA TAILLE ET LE POIDS DES PORTÉES OBTENUES PAR INSÉMINATION ARTIFICIELLE
}

\author{
I. OLLIVIER et C. LEGAULT \\ avec la collaboration technique de B. Dando et L. Gullbault \\ Station centrale de Génétique animale, \\ Cenire national de Recherches zootechniques, 78 -Jouy-en-Josas \\ Institut national de la Recherche agronomique, \\ Centre expérimental de Sélection et d'Insímination porcine, 86 -Rouillé
}

SOMMAIRE

L'effet direct du verrat sur la taille et le poids des portées qu'il engendre a été étudié sur un ensemble de I 327 portées contrôlées à la naissance, de I OIO portées pesées à la naissance et de 906 portées pesées au sevrage. Ces portées sont issues de 30 verrats utilisés de I96r à I964 au Centre expérimental de Sélection et d'Insémination porcine de Rouillé (Vienne). Les effets du verrat, de la période semestrielle et du numéro de portée ont été estimés par la méthode des moindres carrés. Les différences entre verrats et entre périodes semestrielles sont hautement significatives pour la taille de la portée à la naissance. Ces deux facteurs expliquent respectivement 4,9 et $4,4 \mathrm{p}$. roo de la variance totale du caractère. Le poids global de la portée à la naissance, la taille et le poids global de la portée au sevrage ne sont pas significativement influencés par ces facteurs. L'effet du numéro de portée est hautement significatif pour toutes les variables.

\section{INTRODUC'TION}

Un précédent travail (LEgaulit et OlLivier, I965) démontrait que le verrat exerce sur les portées qu'il engendre un effet hautement significatif quant au nombre de porcelets à la naissance, mais non significatif quant au nombre de porcelets sevrés.

L'objet de la présente étude est de compléter l'analyse faite précédemment en considérant un nombre plus important de verrats et en incluant comme variables supplémentaires le poids de la portée à la naissance et au sevrage. 


\section{MATÉRIEL ANIMAL E'T MÉ'THODE D'ANALYSE}

Les données concernent des portées de race Large White obtenues par insémination artificielle de I960 à ig64 dans l'aire d'extension du Centre expérimental de Sélection et d'Insémination porcine de Rouillé (Vienne). Ces portées ont été examinées et, sauf en quelques cas, pesées globalement, une première fois dans un délai de 5 jours après la naissance et une deuxième fois aux environs du sevrage, entre 40 et 64 jours. Dans cette étude, nous avons retenu les 30 verrats ayant un minimum de 10 portées examinées dans un délai de 5 jours après la naissance, ce qui représente un effectif de I 327 portées.

Les poids de portée ont été corrigés en fonction du jour de la pesée de la manière suivante : les portées ont d'abord été classées par nombre de porcelets nés, et, dans chaque classe de taille de portée, la régression du poids de la portée sur l'âge à la première pesée a été calculée. Les poids ont alors été ramenés à o jour. Ensuite les portées ont été classées par nombre de porcelets sevrés, les régres. sions du poids de la portée sur l'âge à la deuxième pesée ont été calculées et les poids ramenés à 52 jours.

Cinq variables ont été considérées dans l'analyse :

variable I : nombre total de porcelets nés (vivants et morts) ; variable 2 : nombre de porcelets vivants à la première pesée ; variable 3 : nombre de porcelets vivants à la seconde pesée; variable 4 : poids de la portée à la naissance (corrigé) ; variable 5 : poids de la portée à 52 jours (corrigé).

Les données ont été classées par verrat (30), par période de mise bas de 6 mois ( 9 périodes allant d'octobre 1960 à décembre 1964 : octobre 1960 -mars 1961 , avril 1961 -septembre $1961 \ldots$, avril I 964 septembre I964, octobre I964-décembre I964) et par numéro de portée (I, 2, 3, 4, 5 et plus). La. méthode des moindres carrés a permis d'estimer les effets de chacun de ces facteurs et de faire les tests statistiques comme indiqué par Legault et Ollivier (I965). Les composantes de la variance ont ensuite été estimées selon la méthode I donnée par HENDERson (I953), en supposant aléatoires les niveaux de chaque facteur. En réalité, les effets "verrat " et " période semestrielle " peuvent être considérés comme aléatoires alors que le numéro de portée est plutôt une variable dont les niveaux sont fixés.

\section{RÉSULTATS}

les figures I et 2 montrent l'accroissement journalier du poids des portées en fonction du nombre de porcelets, d'une part dans les 5 jours qui suivent la naissance et d'autre part entre 40 et 64 jours. Dans le premier cas, les résultats sont irréguliers, car l'accroissement de poids de chaque porcelet est contrebalancé par la mortalité importante qui intervient durant cette période. Dans l'ensemble cependant, il apparaît que l'accroissement journalier de poids passe par un maximum pour les portées de 8,9 et ro porcelets à la naissance et qu'il est voisin de zéro pour les portées de faible effectif et celles d'effectif supérieur à $\mathrm{I} 6$. Au contraire, au voisinage du sevrage, l'accroissement de poids passe de I à $4 \mathrm{~kg}$ assez régulièrement à mesure que l'effectif augmente.

Le tableau I donne les résultats des tests d'interaction. Aucun n'étant significatif, l'analyse a été poursuivie en faisant l'hypothèse de non-interaction entre les trois facteurs "verrat ", " période semestrielle " et " numéro de portée ". Les effets de chaque facteur ont été testés (tab1. 2). Les valeurs de " $F$ » montrent que le facteur le plus important est le "numéro de portée " dont les effets sont hautement significatifs pour les cinq variables. I, a période semestrielle a un effet significatif seulement sur le nombre à la naissance. L, effet direct du verrat est hautement signifi- 

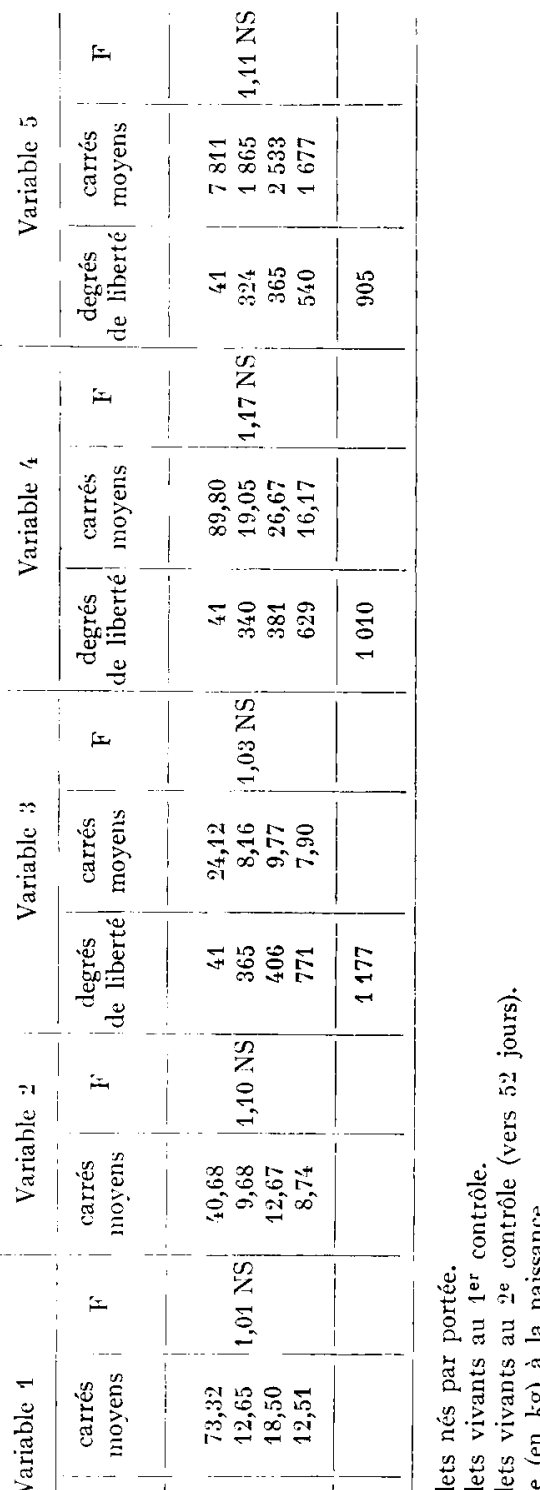

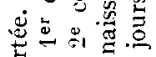

总灵 正䠉

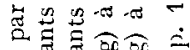

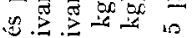

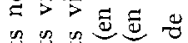

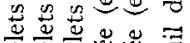

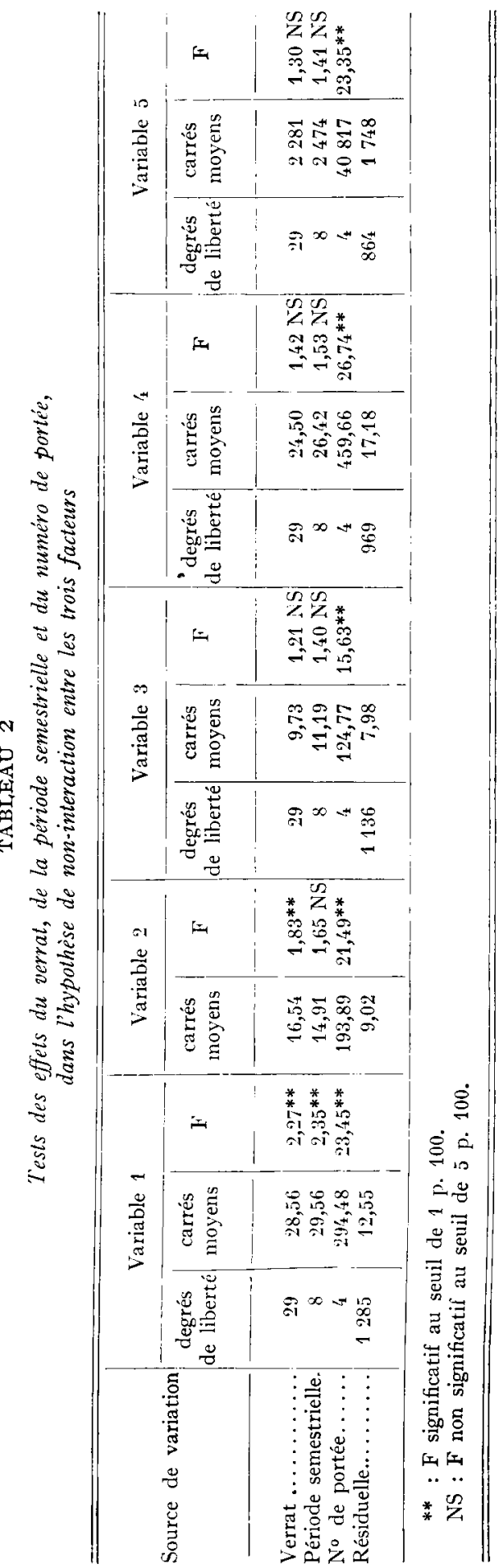


I. OLLIVIER, C. LEGAULT

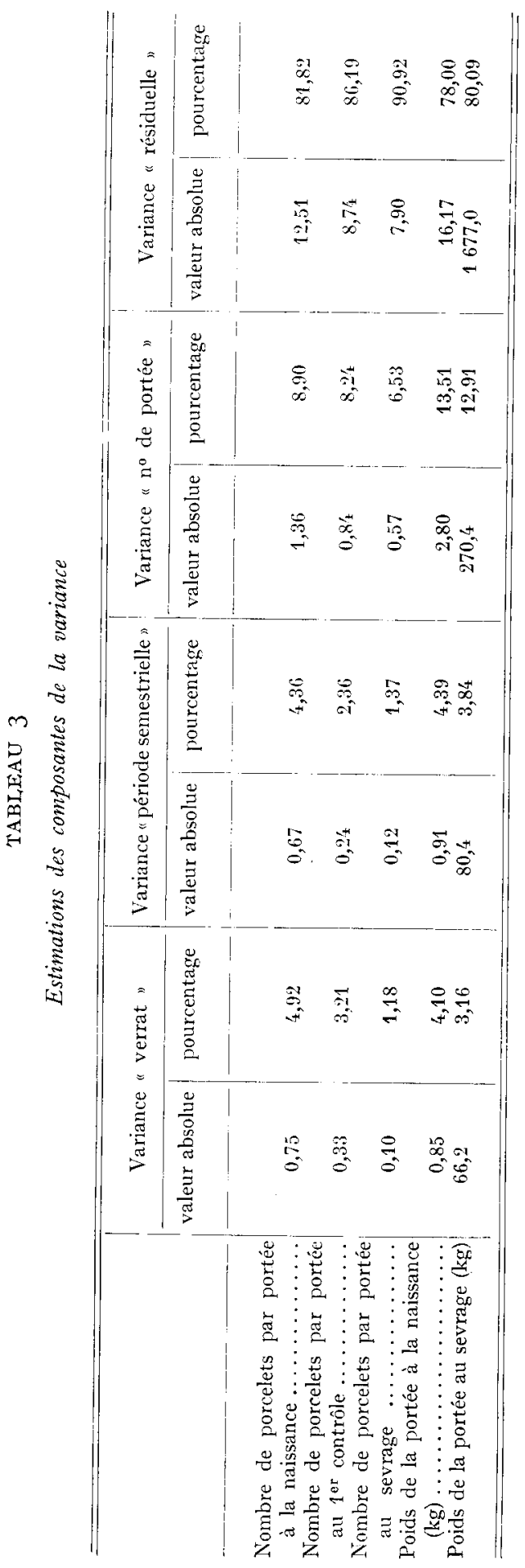


catif quant au nombre de porcelets nés et au nombre de porcelets vivants au premier contrôle, mais non significatif quant au nombre sevré et quant aux poids des portées. Les composantes de la variance sont données dans le tableau 3.

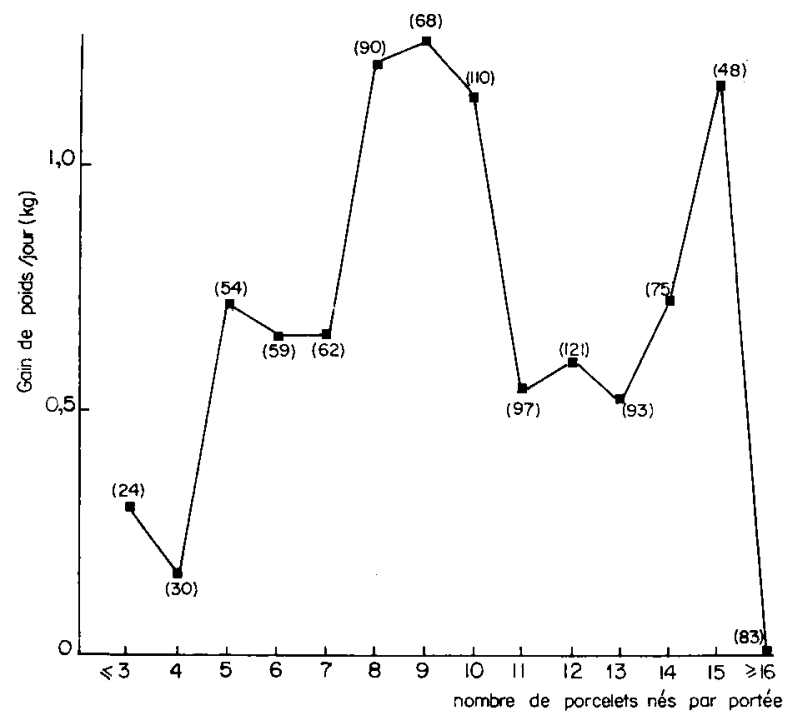

Fig. I. - Accroissement journalier du poids de la portée (entre la naissance et l'ige de 5 jours) en fonction de la laille de la portée d̀ la naissance (effectifs entre parenthises)

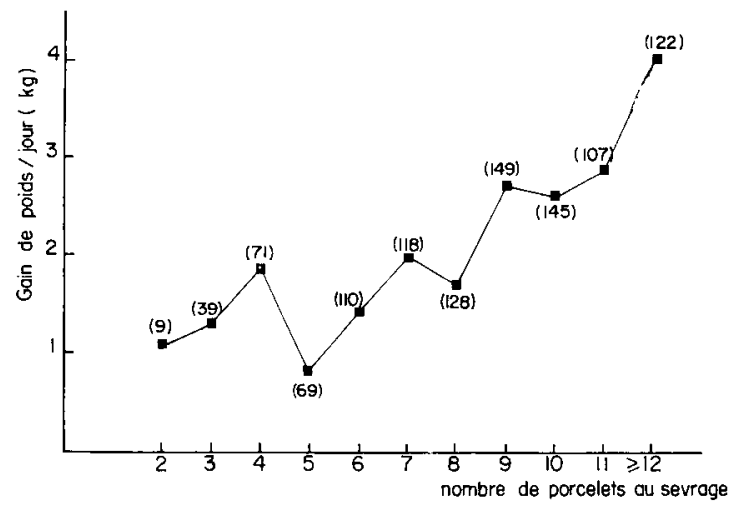

Fic. 2. - Accroissemenl journalier du poids de la porlée (entre l'áge de 40 et de 64 jours) en fonclion de la taille de la portée. (effectifs entre parenthèses)

\section{DISCUSSION ET CONCLUSIONS}

Les résultats ci-dessus appellent la même discussion que celle donnée dans le précédent travail (LEGAULT et OLLIVIER, I965). Il y a cependant des correctifs à apporter et quelques différences à souligner. 
D'abord l'analyse qui vient d'être décrite met en évidence une influence importante de la période semestrielle sur la taille de la portée à la naissance, effet qui n'apparaissait pas dans l'analyse précédente où seule l'année était prise en considération. La figure 3 montre une alternance saisonnière assez régulière, octobre-mars étant le semestre favorable. Des enquêtes statistiques effectuées sur le troupeau français révèlent l'existence, dans les conditions de la monte naturelle, d'une fluctuation

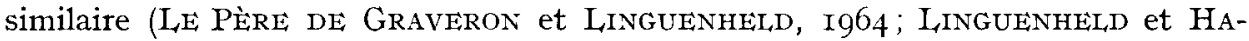
ZARD, I966). Cette variation saisonnière de la prolificité est probablement liée à celle de la fertilité, exprimée en pourcentage de mise-bas par insémination. Ce pourcentage accuse, en effet, une baisse de dix points pendant les mois de juillet, août et

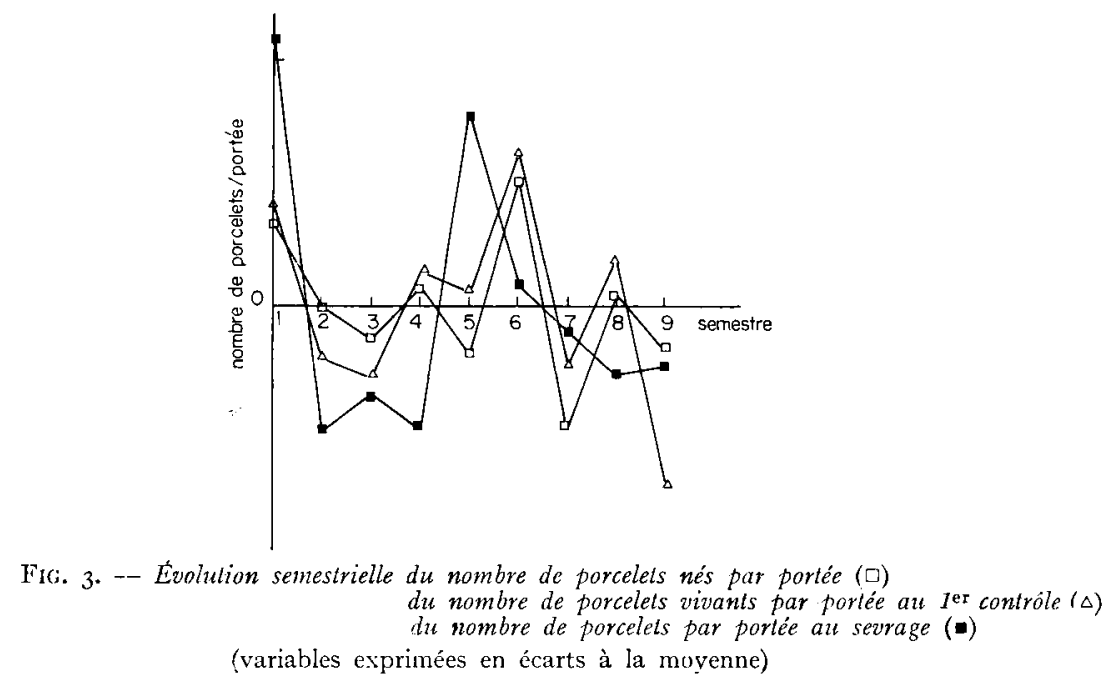

septembre dans le même centre d'insémination (CORTEEL et al., Ig64). A cette variation cyclique se superposent, dans notre échantillon, des variations plus importantes, attribuables à des effets " période particulière ". L'origine de ces variations n'est pas claire. Elles peuvent être liées à des facteurs climatiques. La figure 3 montre également que les effectifs par portée au premier contrôle et au sevrage subissent des variations parallèles, mais à l'opposé de l'effectif à la naissance, c'est le semestre avril-septembre qui est le plus favorable. Les différences entre semestres ne sont cependant pas significatives.

La différence entre le verrat le plus prolifique et le verrat le moins prolifique atteint 5,20 porcelets à la naissance et 2,92 au sevrage. Ces écarts sont plus importants que dans l'étude précédente par suite d'un échantillonnage moins restreint.

Enfin, 1'effet direct du verrat sur le poids des portées n'est pas statistiquement significatif bien que la valeur de « $F$ » soit très proche du seuil de signification à 5 p. Ioo dans le cas du poids de la portée à la naissance. Ceci confirme l'hypothèse que l'influence du verrat s'exerce davantage sur le nombre d'embryons arrivant au terme de la gestation que sur leur poids, un nombre élevé de porcelets nés étant généralement compensé par un poids moyen faible. On sait d'ailleurs que, chez le porc, l'héritabilité du poids individuel à la naissance est trèss voisine de zéro (voir par exemple LEGAUI,T 


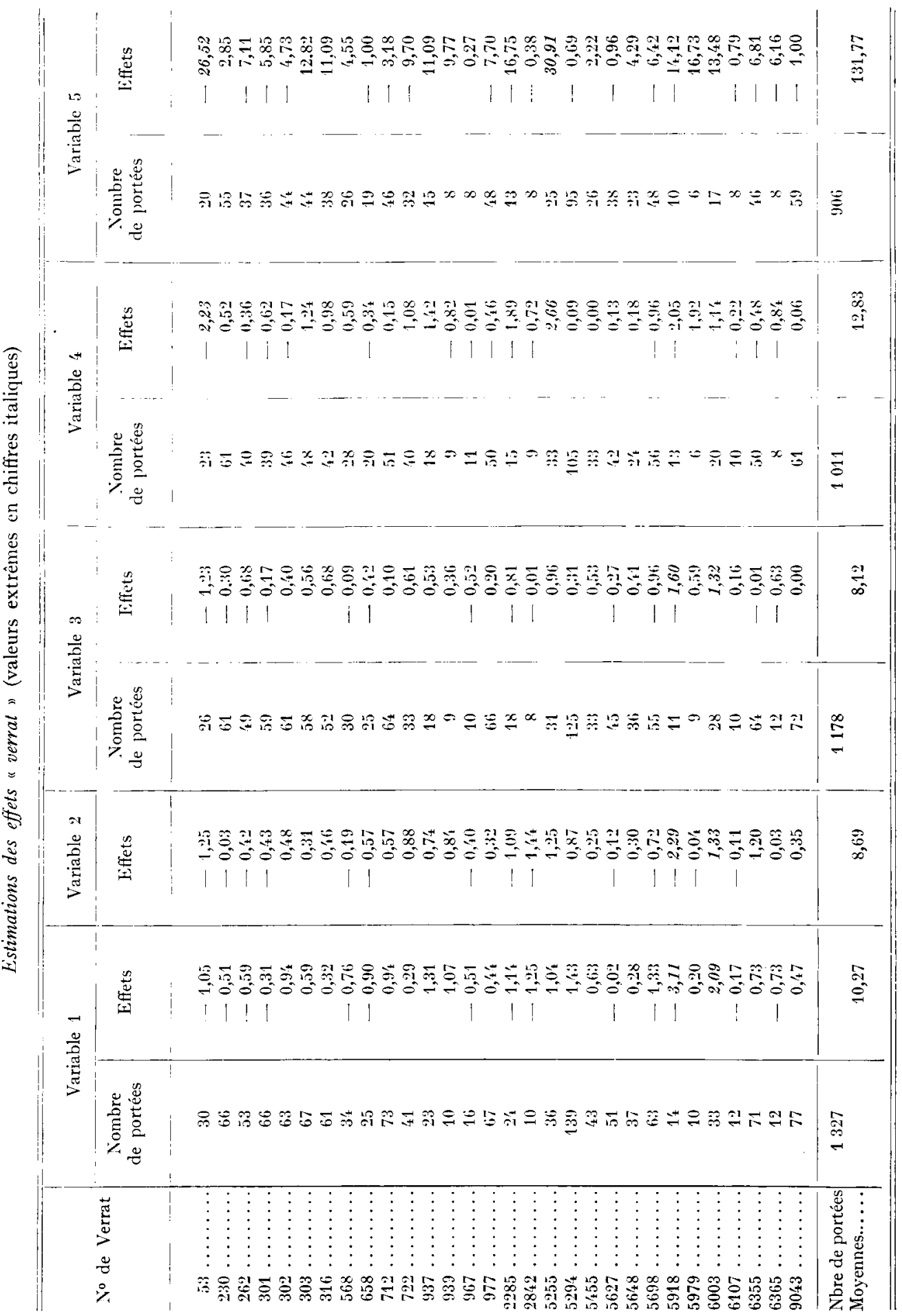

Annales de Zootechnie. - 1967 . 
et Aumaitre, I966). Dans l'ensemble, poids et effectif de portée varient dans le même sens entre verrats, et l'information qu'apporte la pesée n'est pas en rapport avec le supplément de travail qu'une telle opération requiert. De plus, le poids d'une portée est une variable complexe et ses variations dans les premiers jours qui suivent la naissance sont dues à des causes très variées (rusticité et croissance du porcelet, aptitudes maternelles, milieu d'élevage, décisions de l'éleveur, etc.) ce qui rend toute correction difficile en fonction de l'âge.

La part de la variance attribuable au verrat père de la portée avoisine $5 \mathrm{p}$. Ioo pour la taille de la portée à la naissance. On peut donc dire que l'influence du verrat père de la portée est sensiblement plus importante que l'influence génétique du père de la truie dans la détermination de la taille de la portée à la naissance, dont l'héritabilité est de 1'ordre de o,Io. Les deux facteurs verrat (père de la portée) et saison expliquent à eux seuls plus de 9 p. Ioo de la variance du nombre de porcelets par portée. Il est donc important d'en tenir compte dans tout programme de sélection visant à améliorer ce caractère.

\section{Reçu pour publicalion en juin 1967.}

\section{SUMMARY}

INFLUENCE OF THE BOAR ON LITTER SIZE

AND WEIGHT IN ARTIFICIAL INSEMINATION

The effect of the boar on litter size and weight was estimated from a sample of litters born from 30 Large White boars between 1961 and 1964 at RoulLLE experimental Artificial Insemination Centre. The effects of the boar, the six month period of birth and the litter order were estimated by least squares analysis. The differences between boars and between periods were highly significant for litter size at birth. Litter weight at birth, litter size and weight at weaning showed no significant effect of the boar and the period. Litter order had a highly significant effect on all characteristics.

\section{RÉFÉRENCES BIBLIOGRAPHIQUES}

Corteel J. M., Signoret J. P., Du Mesnil du Buisson F., ig64. Variations saisonniéres de la reproduction de la truie et facteurs favorisant l'anœstrus temporaire. Ve Congrès de Reproductionanimale et d'Insémin. arifif. Trente, 3, 536-540.

Henderson C. R. 1953. Estimation of variance and covariance components. Biometrics, 9, 226-252.

LEgault C., Ollivier L., I965. Résultats préliminaires concernant l'influence du verrat sur la taille de la portée en insémination artificielle. Ann. Zootech., 14, 40I-408.

Legault C., Aumaitre A., 1966. Aspects biométriques de la croissance pondérale du porcelet. II. Étude génétique. Ann. Zoolech., 15, 333-34I.

Le Père de Graveron J., Linguenheld R., ig64. Les performances d'élevage d'un échantillon de 70000 truies. Rev. Élev., 19, 629-638.

Linguenheld R., Hazard C., ig66. Performances d'élevage du troupeau porcin français en ig64. Re?. Élev., 21, 77-95. 